\title{
Der Beitrag von Francesco Buzzi zur Entdeckung der «Macula lutea» und der «Fovea centralis» des menschlichen Auges
}

Von Luigi Belloni

Der fünfte Band der 1782 in Mailand veröffentlichten «Opuscoli scelti sulle scienze e sulle arti» enthielt, unter dem Titel Nuove sperienze fatte sull'occhio umano dal sig. Francesco Buzzi Chirurgo Oculista ed Ajutante Chirurgo dello Spedale Maggiore di Milano, einen 9 Seiten $^{1}$ umfassenden Artikel, durch welchen der Name seines Verfassers der Medizingeschichte überliefert werden sollte.

Francesco Buzzi (1751-1805) stammte aus Dervio am Comersee ${ }^{2}$ und war in sehr jungen Jahren als «servente» am Ospedale Maggiore in Mailand angestellt worden, wo er gleichzeitig auch die innere Schule des Hospitals für Anatomie und Chirurgie besuchte. Dies ermöglichte es ihm dann, an demselben Krankenhaus ein höherer Chirurg und Augenarzt zu werden.

In der soeben erwähnten Arbeit aus dem Jahre 1782, beschrieb Buzzi eine Präpariermethode, um direkt am frischen Augapfel den Mechanismus des Sehens zu studieren. Die Methode war darauf ausgerichtet, während der zur Prüfung notwendigen Zeit, die Verdunstung und demzufolge das Erschlaffen des Augapfels zu vermeiden, so daß die Form, die Verbindungen und die Beziehungen zwischen den einzelnen Teilen beibehaiten werden konnten, wodurch es dem Beobachter ermöglicht wurde, an einem an Gesamtheit, Straffheit und natürlicher Gestalt vollständigen Augapfel zu arbeiten. Der vom Fett und von den Muskeln befreite Augapfel wirde, an dem durch eine Pinzette gehaltenen Sehnerv hängend, mehrmals und in geeigneten Zeitabständen in eine Mischung von 6 Teilen geschmolzenen Wachses und 1 Teil Terpentin getaucht, so daß er sich am Ende der Behandlung in einer festen, zwiebelartig geschichteten Umhüllung befand. Es wurden dann zwei Kalotten der Wachsschale entfernt - eine vordere und eine hintere - um die Hornhaut bzw. den Ansatz des Sehnervs mit dem der Hornhaut entgegengesetzten Teil der Sklera bloß zu legen. Daraufhin, und je nach den verschiedenen Experimenten, ging Buzzi dazu über, die Augenhäute einzeln oder gemeinsam zu entfernen und in gewissen Fällen einige oder alle Säfte abzusondern. Der Verfasser ist in seiner Darstellung derart bescheiden, daß 
ich mich gezwungen sehe, die von ihm mit dieser Methode durchgeführten Experimente zu übergehen, da ich sonst seine Arbeit übersetzen würde, anstatt sie zusammenzufassen. Ich möchte hier nur hervorheben, daß er aus diesen Erfahrungen den Schluß zieht, die Netzhaut sei jener Teil des Auges, auf dem sich das Bild des Gegenstandes umgekehrt abzeichnet.

Hier werden wir uns hingegen etwas länger über das VII. Experiment aufhalten, das er an sechs mit der üblichen Methode präparierten und zu optischen Kammern gemachten menschlichen Augen durchführte. Nachdem Buzzi am hinteren Pol die übliche Abhebung der Leder-, Ader- und Netzhaut vollendet hatte, bringt er an Stelle der letzteren ein sehr dünnes, halbdurchsichtiges, farbiges Seidenband an, und legt ein Glas darüber, worauf er das Bild des Objektes, das dem Band selbst entsprechend gefärbt ist («gelb, grün, blau, weinrot, rot und schwarz») sieht. Demzufolge denkt er «eine bis jetzt sehr diskutierte Frage endgültiglösen zu können, d. h., daß die Vergilbung der Netzhaut der wahre Grund ist, weshalb der manchmal an hartnäckiger Gelbsucht leidende Mensch die äußeren Gegenstände gelb gefärbt sieht». Zur Unterstützung dieser Behauptung führt er den an den Augen zweier Gelbsüchtiger beobachteten anatomischen Befund an ${ }^{3}$.

Im ersten Gelbsüchtigen, der «in den letzten Tagen seines Lebens ... alle äußeren Gegenstände gelb gefärbt sah», bemerkte Buzzi, daß «die Säfte des Auges durchsichtig waren und die Netzhaut, besonders gegen den Hintergrund hin, eine entschieden gelbe Färbung aufwies, weil das die Blut- und Lymphgefäße umgebende Zellgewebe an dieser Stelle in größerer Menge vorliegt. Ja, an einer seitlich des Sehnervs liegenden Stelle sieht man, auch in gesundem Zustand, immer dieselbe, aber sehr blaß gelb gefärbte Netzhaut». ${ }^{4}$

Am zweiten Gelbsüchtigen, der hingegen «die Gegenstände niemals nicht einmal in den letzten Tagen seines Lebens - gelb gefärbt gesehen hatte», erkannte Buzzi, daß «die Netzhaut an einigen kaum sichtbaren Stellen ihrer konvexen Seite hellgelb gefärbt war; und nur jene Stelle, an der die Netzhaut - wie soeben erwähnt - natürlich gelb ist ${ }^{5}$, war durch ein entschieden stärkeres Gelb gefärbt».

Zwei Jahre später, 1784, wies Buzzi anläßlich seiner eingehenden Beschreibung der Augenanatomie eines Falles von menschlichem Albinismus nochmals auf den Befund der Macula lutea hin: «Die Netzhaut zeigte keinerlei Besonderheit; sie erschien nur viel weißer und dünner als gewöhnlich. Jene gelbliche Stelle neben dem Sehnerv ${ }^{6}$, worauf ich bereits in meinen neuen Erfahrungen an dem zu optischer Kammer gemachten menschlichen Auge hinwies, wurde durch eine hellgelbe Farbe beträchtlich hervorgehoben». ${ }^{7}$ 
Es besteht demnach kein Zweifel, daß «jene gelbliche Stelle neben dem Sehnerv», die Buzzi nicht nur in drei abnormen Fällen, sondern «auch in gesundem Zustand» beobachtete, für ihn einen normalen Befund darstellte. Ihm gebührt also der erste veröffentlichte Hinweis der Macula lutea: Der Nachdruck, womit Buzzi immer wieder auf diesen Befund zurückkommt, ersetzt die Kürze seiner Beschreibung.

Um seinen Studenten die Netzhaut zu demonstrieren, präparierte Samuel Thomas Soemmerring (1755-1830), Professor der Anatomie und Physiologie an der Mainzer Universität, am 27. Januar 1791, «oculos recentissimos, perfecte adhuc pellucidos, summeque turgidos», die er der Leiche eines wenige Stunden vorher im Rhein ertrunkenen Jünglings entnommen hatte. Während er am ersten der beiden Augen arbeitete, indem er es «suspensa manu in liquore idoneo» hielt, fiel ihm am hinteren Teil der Netzhaut, die, dank der Prallheit des Augapfels, vollkommen gestrafft war und nicht die kleinste Falte aufwies, ein sonderbarer Befund auf. Mit größter Überraschung beobachtete er «maculam rotundam luteam», und zwar so ausgeprägt, daß er sofort die Möglichkeit eines technischen Artefaktes ausschloß. Bei näherer Betrachtung bemerkte er in der Mitte der Macula ein kleines Loch, das genau den Mittelpunkt der Netzhaut einnahm («in medio eius foraminulum veri centri retinae locum occupans»). Nachdem er diese Befunde auch am entgegengesetzten Auge festgestellt hatte, demonstrierte er sie öffentlich im Hörsaal und kündigte die Entdeckung kurz in der Einführung seiner Abhandlung Vom Baue des menschlichen Körpers («De corporis humani fabrica»), deren Veröffentlichung gerade 1791 in Frankfurt eingeleitet wurde, an.

Erkundigungen, die Soemmerring bei Freunden und Verwandten des Ertrunkenen einholte, erlaubten es ihm auszuschließen, daß der Jüngling an Sehstörungen gelitten hatte und so «rem novam ulterius meditando» wollte er sich von der Beständigkeit der Befunde versichern. Zu diesem Zwecke öffnete er mindestens 50 Augen und ließ zur Kontrolle durch seine Schüler Sommer in Brunswick, Behrends in Frankfurt, Michaelis in Göttingen, Creve in Trier, Wenzel und Schenzer in Mainz, Nachprüfungen durchführen, deren Ergebnisse sämtlich positiv ausfielen.

Am 1.August 1795 gab Soemmerring, sich auf diese Bestätigungen stützend, bei der K. Gesellschaft der Wissenschaften zu Göttingen seine Entdeckung bekannt: De foramine centrali limbo luteo cincto retinae huma$n a e^{8}$. 
In ihrer grundsätzlichen Durchführung - teilweise und zentripetale Abhebung der Augenhäute - stimmt die von Soemmerring angewandte Präparationstechnik mit derjenigen von Buzzi überein: Um die Straffheit des Augapfels beizubehalten, bedient er sich, anstatt des Wachsüberzuges, der schon erwähnten Immersion «in liquore idoneo», und unter diesen Bedingungen geht er dazu über, Kalotten von Sklera und Chorioides vorsichtig vom hinteren Pol zu entfernen, um den der Hornhaut entgegengesetzten Teil der Netzhaut bloßzulegen. Da erschien die Macula lutea mit einem tief schwarzen, vollkommen runden Fleckchen in der Mitte, das von Soemmerring fälschlich als ein kleines Loch ausgelegt wurde: die Macula lutea erhält demzufolge den Namen «luteum limbum foraminis centralis». Ich übergehe hier die vom Verfasser aufgestellten physiologischen Hypothesen, zu denen er geführt wurde, von der Voraussetzung ausgehend, daß im Mittelpunkt der Netzhaut ein kleines Loch vorläge.

Wenige Monate bevor Soemmerring seine Arbeit der K. Gesellschaft der Wissenschaften zu Göttingen mitteilte, gab er sein Manuskript einem jungen Arzte aus Parma, Giovanni Rasori (1766-1837) ${ }^{9}$, zu lesen. Dieser sollte in den künftigen Jahrzehnten auf die italienische und vorwiegend auf die Mailänder Medizin einen Einfluß allerersten Ranges ausüben ${ }^{10}$. Als Schüler der Anatomiker Michele Girardi (1731-97) in Parma, Michelangelo Gianetti (1743-96) in Florenz und Antonio Scarpa (1752-1832) in Pavia, war Rasori damals für die chirurgische Laufbahn bestimmt und bezog ein Stipendium der Regierung von Parma, wodurch ihm gegen Ende 1793 die Möglichkeit geboten wurde, eine Studienreise nach London anzutreten und dann seinen dortigen Aufenthalt bis zum Februar 1795 auszudehnen. Auf der Rückreise durch Deutschland kam er nach Frankfurt, «wo er sich mehrere Tage lang mit Soemmerring aus Mainz aufhielt, welcher ihn derart achtete und liebte, daß er ihm über ein unveröffentlichtes Manuskript berichtete, das eine von ihm am Auge gemachte Entdeckung enthielt». ${ }^{11}$

Nach It alien zurückgekehrt, ließ sich Rasori in Mailand nieder, wo er das Stipendium seiner Regierung weiterhin bezog «mit dem Auftrag, sich unter der Leitung von [Pietro] Magistretti [(1765-1837)] ${ }^{12}$, der damals als ein in seinem Fach sehr tüchtiger Mann galt, in den Augenoperationen zu üben und zu vervollkommnen». Rasori ließ es sich deshalb nicht entgehen, die Nachricht über eine neue Entdeckung am Auge sofort bekannt zu machen: Sopra una nuova scoperta nell'occhio ist eben der Titel des von ihm an Giovanni Battista Monteggia (1762-1815) gerichteten Briefes ${ }^{13}$, der noch in 
demselben Jahre 1795 in zwei Mailänder Zeitschriften veröffentlicht wurde, worunter die «Opuscoli scelti», in welchen $13 \mathrm{bzw} .11 \mathrm{~J}$ ahre früher die beiden Arbeiten von Buzzi mit der Beschreibung der Macula lutea erschienen waren.

Die Veröffentlichung des Briefes von Rasori, der im wesentlichen eine Zusammenfassung der Arbeit von Soemmerring darstellt, konnte natürlich Buzzi nicht gleichgültig lassen: Tatsächlich entgegnete er diesem Brief, indem er sich aber - offenbar auf Grund politisch-gerichtlicher Angelegenheiten, in die er verwickelt war - hinter einem Decknamen versteckte. So erschien in denselben Zeitschriften, die den Brief Rasoris verbreitet hatten, die Entgegnung: Lettera del dott. Paolo Antonio Venini al sig. dott. A.C. medico in Milano ${ }^{14}$, die nicht nur einen Anspruch zu Gunsten Buzzis geltend macht, sondern auch die Beschreibung neuer, von ihm durchgeführter Experimente enthält, die vor allem darauf abzielten, zu beweisen, daß sich Soemmerring geirrt hatte, als er den «halbdurchsichtigen Punkt» als kleines Loch beschrieb. Deshalb hat Buzzi auch das Verdienst, als erster jene äußerst starke Verdünnung der Netzhaut erkannt zu haben, woraus die «Fovea centralis» hervorgeht:

\footnotetext{
«An einem von den Muskeln wohl befreiten Auge wird fast die Hälfte des hinteren Teiles der Sklera und der entsprechenden Chorioides abgehoben, indem man sowohl die Netzhaut als auch den Sehnerv unberührt läßt. Daraufhin übt man auf den Augapfel einen Druck aus, damit sich die Netzhaut spannt und sich in jenem Teil des gelben Flecks strafft; dieser Druck verursacht die Glättung der Falte, die den gelben Fleck durchzieht und so erscheint etwa in seiner Mitte ein bald runder, bald ovaler, mehr oder weniger großer, halbdurchsichtiger Punkt. Dieser halbdurchsichtige Punkt ist das, was der Mainzer Professor als ein kleines, undurchlässiges Loch bezeichnet.»

«Um zu prüfen, ob dieser halbdurchsichtige Punkt offen sei, führte Buzzi selbst ein dünnes Röhrchen zwischen die Netzhaut und die Arachnoides in größtmöglichster Entfernung von der Macula lutea ein. Daraufhin blies er mit allmählich fortschreitender Stärke in das Röhrchen, um die Netzhaut allein aufzublasen und zu sehen, ob aus diesem halbdurchsichtigen Punkt Luft ausströmte; aber auch nach mehrfacher Wiederholung des Experiments konnte er nie eine Ausströmung der Luft feststellen; hingegen erkannte er noch deutlicher, daß die Netzhaut an diesem Punkte dünner und durchscheinender ist.» ${ }^{15}$
}

Die von Soemmerring am 1.August 1795 bei der K. Gesellschaft der Wissenschaften zu Göttingen gehaltene Mitteilung wurde erst 1799 in den entsprechenden akademischen Sitzungsberichten «in extenso» publiziert; aber in wissenschaftlichen Kreisen wurde sie unmittelbar durch eine, am 31. August 1795 in einer in Verbindung mit derselben Gesellschaft publizierten Zeitschrift erschienene Zusammenfassung bekannt ${ }^{16}$. Diese Zusammen- 
fassung wurde von anderen Zeitschriften wieder aufgenommen und erschien auch in Nummer 14,17 des «Journal der Erfindungen, Theorien und Widersprüche in der Natur- und Arzneiwissenschaft» (Gotha 1796), wo der vollständige italienische Text des Mailand, den 10. Januar 1796 datierten Schreibens: Lettera del dott. Paolo Antonio Venini al sig. dott. A. C. medico in Milano, folgt ${ }^{18}$. Nach Beendigung des Briefes, verspricht der Verfasser, im darauffolgenden Heft einen eingehenden, von einem deutschen Arzte geschriebenen und sich auf originelle Erfahrungen stützenden Artikel zu publizieren.

Das nachfolgende, ebenfalls 1796 erschienene Heft 15 enthält tatsächlich einen langen, Braunschweig, im Januar 1796 datierten, und vom Militärarzt Dr. Philip Michaelis ${ }^{19}$ unterzeichneten Brief ${ }^{20}$. Er erzählt, daß er im Sommer 1792, während eines Aufenthaltes in Wien, einen Brief erhielt, worin ihm Soemmerring Nachricht der von ihm entdeckten Befunde gab; da er sie «aber damals noch nicht für den natürlichen Bau hielt», forderte er ihn auf, Nachprüfungen über diesen Gegenstand durchzuführen. Michaelis folgte der Aufforderung und machte sich in der Anatomie des Wiener Krankenhauses sofort an die Arbeit: Gleich bei der Überprüfung des ersten Auges erschienen ihm jene Befunde mit derartiger Deutlichkeit, daß er sich nicht erklären konnte, wie sie den vorausgegangenen Forschern hatten entgehen können. Da er auch in den nachfolgenden untersuchten Augen dieselben Befunde feststellte, schloß er daraus, daß es sich um normale, und nicht von einer krankhaften Veränderung verursachte Bildungen handelte.

Im Oktober desselben Jahres traf Michaelis in Wien mit Soemmerring zusammen und teilte seinem Lehrer diese Ergebnisse mit: Dieser hatte jedoch anscheinend in der Zwischenzeit keine weiteren genaueren Untersuchungen darüber durchgeführt. Bald darauf war jedoch Michaelis gezwungen, seine Forschungen zu unterbrechen. Er nahm sie erst später in Pavia ${ }^{21}$ und in Mailand, wo er sich einige Zeit aufhielt, wieder auf. In diesem Zusammenhang sagte er: «Eine so günstige Gelegenheit konnte ich mir bestimmt nicht entgehen lassen, da mir im Spital dieser letzteren Stadt täglich mindestens 10 Leichen von verschiedenstem Alter zur Verfügung standen». Und so tritt in der Geschichte der Macula lutea das Mailänder Ospedale Maggiore wieder in den Vordergrund.

Noch im Jahre 1796 erschien im Heft 17 des «Journal der Erfindungen» ein neuer, Haarburg, den 22. April 1796 datierter Brief von Michaelis ${ }^{22}$, worin dieser mehrere Themen behandelt. Uns interessiert jener Teil, in dem Michaelis den Brief (Lettera) von Venini ${ }^{23}$ erörtert, der im Heft 14 derselben 
Zeitschrift neugedruckt worden war, und zwar besonders dessen Anfang ${ }^{24}$ : «Dasselbe Stück Ihres Journals enthält einen Brief aus Mailand über den gelben Fleck und das Loch in der Retina. Es freuet mich, daß die Beobachtungen von Buzzi mit den meinigen oft wirklich übereinstimmen.»

\section{Anmerkungen}

1 S. 87-95.

${ }^{2}$ Weiteres über Buzzi und seine anatomischen und klinischen Schriften ist in meiner Arbeit Francesco Buzzi e la scoperta della macula lutea, in «L'Ospedale Maggiore», 44 (1956), 223-234, enthalten. Ein kurzer Hinweis von mir auf Buzzi und seine Forschungen über die Netzhaut findet sich auch in «Storia di Milano» della Fondazione Treccani degli Alfieri, 16 (1962), 942-943.

${ }^{3}$ Fünfzehn Jahre später erschien ein Auszug in deutscher Sprache des Artikels von Buzzi, der sich auf die Augenbefunde der beiden Gelbsüchtigen bezieht: Section, zweier, an der Gelbsucht Verstorbenen, die besonders in Rücksicht auf die Beschaffenheit der Augen gemacht wurde von Franz Buzzi, in «Italienische medicinisch-chirurgische Bibliothek oder Übersetzungen und Auszüge aus den neuern Schriften italienischer Ärzte und Wundärzte», III. Bd., II.Stück (1797), S.96-98. Die Zeitschrift wurde in Leipzig herausgegeben durch den Arzt Carl Weigel (1769-1845), der folgende Anmerkung beifügte: «Die Entdeckung, welche der, um die Medicin so sehr verdiente, Hr. Hofrath Sömmering vergangenes Jahr machte, war jenseits der Alpen schon vor fünfzehn Jahren gemacht. Indess blieb sie in Teutschland unbekannt, und teutsche Ärzte sind ihm, so wie für viele andre Belehrungen Dank schuldig.»

${ }^{4}$ Die Kursivschrift ist von uns.

${ }^{5}$ Die Kursivschrift ist von uns.

${ }^{6}$ Die Kursivschrift ist von uns.

7 «Opuscoli scelti sulle scienze e sulle arti», 7 (1784), 84-85.

${ }^{8} \mathrm{~S}$. Th. Soemmerring, De foramine centrali limbo luteo cincto retinae humanae, in «Commentationes Societatis Regiae Scientiarum Gottingensis ad A. MDCCXCV.-XCVIII.», 13 (1799), 3-13, u. 2 Tafeln mit 6 Abb. in doppelter Ausführung. Eine Zusammenfassung der Arbeit war kurz nach seiner Mitteilung erschienen in «Göttingische Anzeigen von gelehrten Sachen unter der Aufsicht der Königl. Gesellschaft der Wissenschaften», 2 (1795), 1401-1402 (140. Stück. Den 31. August 1795.).

${ }^{9}$ G. del Chiappa, Della vita di G.Rasori, Mailand, 1838.

10 Antonio Cazzaniga, La grande crisi della medicina italiana nel primo Ottocento, Mailand, 1951, 122 Seiten. Diese wichtige kritische Arbeit wurde in Fortsetzungen in den Bänden 4, 5 , 6 (1948-1950) der Zeitschrift «Castalia» veröffentlicht.

${ }^{11}$ G. del Chiappa, 1.c., S. 17.

12 Luigi Belloni, Immatrikulations- und Testaturkunde des Tessiner Augenarztes Pietro Magistretti (1765-1837), in «Gesnerus», 5 (1948), 34-42.

${ }^{13}$ Lettera del dott. G. Rasori al sig. G. B. Monteggia sopra una nuova scoperta nell'occhio: del prof. Soemmering di Magonza, in «Nuovo giornale della più recente letteratura medico-chirurgica 
d'Europa» (Mailand), 9 (1795), 209-214; und in «Opuscoli scelti sulle scienze e sulle arti» (Mailand), 18 (1795), 376-379.

1" Lettera del dott. Paolo Antonio Venini al sig. dott. A. C. medico in Milano, in «Nuovo giornale della più recente letteratura medico-chirurgica d'Europas, 9 (1795), 370-376; und unter dem Titel: Lettera del Sig. Dott. Paolo Antonio Venini al Sig. Dott. A.C. medico in Milano. Sulla scoperta del Sig. Soemmering nell'occhio umano, in «Opuscoli scelti sulle seienze e sulle arti», 19 (1796), 22-26.

15 Die Kursivschrift ist von uns.

16. Vgl. Anmerkung 8 .

17 S. 117-119.

$18 \mathrm{~S} .119-127$.

19. «ehemal. Hannöver. Feldarzt, jetzt Garnisonsarzt zu Haarburg».

${ }_{20} \mathrm{Ph}$. Michaelis, Über einen gelben Fleck und ein Loch in der Nervenhaut des menschlichen Auges, in «Journal der Erfindungen, Theorien und Widersprïche in der Natur- und Arzneiwissenschaft» (Gotha), XV.Stück (1796), 3-17.

${ }^{21}$ Luigi Belloni, Charles Bonnet et Vincenzo Malacarne sur le cervelet siège de l'âme et sur l'impression basilaire du crâne dans le crétinisme, in «Gesnerus», 34 (1977), 69-81, vgl. 72-73 und Anmerkung 29; Charles Bonnet e Vincenzo Malacarne sul cervelletto quale sede dell'anima e sulla impressione basilare del cranio nel cretinismo, in «Physis», 19 (1977), 111-160; vgl. 114 u. Anmerkung 13.

22 Ph. Michaelis, Kurze Bemerkungen, in «Journal der Erfindungen, Theorien und Widersprüche in der Natur- und Arzneiwissenschaft» (Gotha), XVII.Stück (1796), 121-133.

23 Vgl. die mit Anmerkungsnummer 18 vermerkte Stelle im Text.

24 S.127-128.

\section{Zusammenfassung}

Darstellung der vom Chirurg und Augenarzt Francesco Buzzi (1751-1805) in der Anatomie des Ospedale Maggiore in Mailand gemachten Beobachtungen über die Macula lutea (1782) und die Fovea centralis (1795) in der Netzhaut des menschlichen Auges.

\section{Summary}

This article is concerned with the observations on the macula lutea (1782) and the fovea centralis (1795) in the retina of the human eye, made by the surgeon and ophthalmologist Francesco Buzzi (1751-1805) at the Ospedale Maggiore in Milan.

Prof. Dr. med. Luigi Belloni

Istituto di Storia della Medicina

Università degli Studi

Via Festa del Perdono, 7

I-20122 Milano 